\title{
Investigation of hyperspectral image pixel signatures by the empirical mode decomposition method
}

\author{
P A Pakhomov ${ }^{1}$, A V Borusyak ${ }^{1}$ and V E Turlapov ${ }^{1}$ \\ ${ }^{1}$ National Research Lobachevsky State University of Nizhny Novgorod, Gagarin Avenue 23, \\ Nizhny Novgorod, Russia, 603950
}

\begin{abstract}
The signature of hyperspectral image (HSI) pixels and their decomposition into empirical modes (EM) and low-frequency residuals are investigated. On the basis of estimates related to the EM-decomposition method, the possibility of switching from a 2-byte representation of the values of the HIS-signature to a 1-byte one is examined using the example of the Moffett Field from the AVIRIS spectrometer. It is revealed that the localization of the minimum window sizes for the first EM is correlated with the localization of the significant influence of the atmosphere; the first low-frequency residues have a fairly high correlation coefficient with the signature and the first 2 of them and their EM are most interesting for use; 50 of the 224 HIS-channels are noisy and can be excluded from consideration; EM with practically no loss of accuracy can be reduced to a 1-byte representation. The management of the classification capabilities of signatures by changing the threshold value of the correlation coefficient with the sample, as well as the application of the 1st and 2nd low-frequency residues in place of the signature, was studied. Classification capabilities of signatures in a 1byte representation are almost equivalent to a 2-byte one, which makes it possible to put a signature with 1-byte representation as the object of compression. For the wavelet decomposition of the HSI data array, in combination with a 1-byte representation, a nearlossless compression ratio of 6.65 is obtained.
\end{abstract}

\section{Introduction}

Hyperspectral image (HSI) compression is the central task in the processing of such images. The first technological stage, where this task was of vital importance, was the transmission of Earth remote sensing (ERS) data to Earth, since it was not practicable to store and fully process such large amounts of data onboard a spacecraft/aircraft. Thus, in [1] published in 1997, the algorithm of Context-Based Adaptive Lossless Image Coding (CALIC) was proposed. This algorithm was two-dimensional, i.e. it compressed images channel-by-channel and, therefore, it was called 2D-CALIC. By that time, it had been shown that the classified context adaptive prediction implemented in the adaptive selection of adaptive predictors (ASAP) and adaptive combination of adaptive predictors (ACAP) methods yielded good results. The ACAP method was equipped with three-dimensional predictors obtained by learning based on land data. Both methods were applied in the well-known Airborne Visible/Infrared Imaging Spectrometer (AVIRIS), where a data density of 6-5 bit/pixel was achieved. Another popular approach is based on the development of nonlinear predictors. This was implemented in the LOCO-I algorithm, standardized as JPEG-LS [2], and in the above-mentioned 2D-CALIC [1], which was soon upgraded by the authors to 3D and was called 3D-CALIC [3]. In the 2004 publication [4], an optimized version of the 3D-CALIC method, called M-CALIC (M-multiband), was proposed. It was based on the multi- 
channel nature of context-based prediction. The transition from the lossless compression algorithm to the near-lossless one in the M-CALIC method was performed in accordance with [5] by applying the sampling of the values with a step $(2 \delta+1)$, where $\delta$ is the permissible rounding error for pixel values.

The choice of the preferable type of compression very much depends on the applications where the HSI thus stored will be used. The papers on the interpretation of hyperspectral aerospace measurements for diagnosing the state of natural and technogenic objects, coastal waters, and crop areas [6], [7], [8] underline the importance of a qualitative improvement in the channel resolution in the HSI for precise classification of the type and state of the objects being observed, and note that these problems cannot be solved by means of multispectral images alone. At the same time, the high correlation of neighboring channels cannot be seen as a reason for excluding one of these channels as uninformative because the entire small fraction of the differing information is necessary to accurately determine the object of interest or its state.

One of the first approaches in the development of compression algorithms for HSI is to optimize the number of spectral channels while preserving the information value of hyperspectral imaging. This approach is reflected both in domestic [9] and in foreign sources [10], [11]. By the early 2000s, two main lines of research were defined to address the problem of optimizing the number of HSI channels [10]: 1) Feature Extraction; 2) Band Selection. With the possible exception of [12], these two lines of research have in common the possibility of detecting the most informative channels at the initial stage by the projective optimization method. In this method, the optimal approach is to project the initial HSI $X$ of the size $n \times N$ ( $n$ is the number of channels, $N$ is the number of pixels per channel) into a new HSI $Y$ of a smaller size $m \times N(m<n)$, which maximizes the projection index $J=J(Y)$, where $Y=A^{T} X$, and $A$ is an $n \times m$ matrix. Usually, the value of the index is estimated through the spectral data variance by the principal component method [9], [11], and the matrix $A$ is formed from the columns (eigenvectors) of the covariance matrix of the original data. Sometimes, after applying the principal component method, the method of independent components, well-proven on non-Gaussian distributions, can be applied [9],[13].

The divergence of information $J$ between two pixels of the HSI was chosen as a criterion in [14] for the synthesis of images reflecting the spatial distribution of the amplitude ratios of like pixels in different channels. Also of interest is the integral estimate for the amount of information proposed in [9] taking into account the signal-to-noise ratio.

In a series of works of 2008-2013 [15], a three-stage algorithm for lossless HSI compression was proposed, including:

1) Taking into account the relationship between HSI channels by calculating the correlation, constructing a linear predictor of the next channel value from the previous one, and forming arrays of deviations of the value of the next channel from the predicted one.

2) Forming an auxiliary data structure for storing unique pairs of groups of element values in a 1byte representation, as well as pointers to these groups.

3) Compression of the data obtained after the transformations by means of a standard entropy algorithm by processing the generated auxiliary data structures.

An approximately $40 \%$ gain in compression was achieved compared to JPEG-LS. It was established that about $45 \%$ of the gain was obtained by using a 1-byte representation of deviations from the predicted values; $15-26 \%$ of the gain was obtained due to the reordering of the channels during compression.

In a series of papers published in 2009-2016 [16],[17],[18],[19] a method of hierarchical lossless compression for the purpose of storing HSI was constructed. The following requirements for the method were formulated: 1) the possibility of compression of multi-channel images; 2) quick access to fragments of compressed images at various scales; 3) low computational complexity of decompression; 4) strict error control; 5) high efficiency in "no-error" mode and with small errors; 6) use of interchannel dependencies; 7) quick access to the specified components of compressed images at various scales; 8) compression of 16-bit images.

The following dependencies on the channel number are investigated and used for compression: 1) the correlation coefficient with the next channel; 2) the channel average $E$; 3) the difference between 
the minimum and maximum in the channel; 4) channel variance $D$. A method of "common reference components" for channel groups in combination with the "sliding approximation" method within the group is proposed, which permits to independently compress groups of neighboring channels. The best achieved value of lossless compression ratio was about 3.6; the recommended number of hierarchy levels was 4 . The nature and the range of value changes in the channel $\Delta X_{i}=\max _{k}\left(x_{k i}\right)-\min _{k}\left(x_{k i}\right)$ were shown depending on the channel number $i$. Thus, for the image "Urban and Mixed Environment" (SpecTIR spectrometer), the maximum value of the changes $\Delta X$ was about 32000 , while for the "Cuprite-1" image (AVIRIS) - about 12000. In this case, the change curves $\Delta X_{i}, E\left(X_{i}\right), D\left(X_{i}\right)$ look more informative for object classification than the correlation ratio curve.

The results of the research into the methods of lossless HSI compression as of 2013 were fixed in the relevant standards [20], [21]. In particular, the values of the lossless compression ratio of the order of 4-5 were stated as very high figures for HSI.

The research performed during the last decade into the role of noise in the compression of HSI, including its role in near-lossless compression and compression with losses, is covered in a series of publications [22], [23], [24]. It was found that: 1) the noise in the hyperspectral images is signaldependent and is almost uncorrelated spatially; 2) the noise parameters in adjacent channels are generally close, although the overall range of variation of the signal-to-noise ratio over hyperspectral images is very wide; 3 ) in almost all hyperspectral images the quality of approximately 80-85 percent of the total number of channels is close to ideal, that is, the PSNR is close to or above $35 \mathrm{~dB}$.

In this situation, it would be of interest to explore some universal methods capable of analyzing the signatures of both individual pixels and their integral forms, in terms of classification with respect to object and background or for detection the presence of noise, as the classification of the object and the background or for the presence of noise detection.

\section{Description of the empirical mode decomposition method}

In our opinion, one can use for the above purposes the method of empirical mode decomposition (EMD). The empirical mode decomposition method was published in 1998 [25], then it was adapted in 2008 for images [26] and is currently applied for a number of image processing tasks [27]. In particular, it can also be applied for hyperspectral images to decompose the signature of an image pixel, similar to a signal, into intrinsic modes having a space/time-varying periodicity. Relying on this property, we want to isolate the highest-frequency part of the signature, which by its nature can represent noise, and also allocate channels (channel groups) that determine individual attributes of the object class specified by the sample pixel.

The decomposition into empirical modes is based on the following assumptions for the signal: 1) the signal has at least two extrema: one maximum and one minimum; 2) the characteristic time scale is determined by the interval between the extrema; 3 ) if the data is completely devoid of extrema (trend), but may contain inflection points, then the signal can be divided into parts in order to reveal the extrema.

The time interval between successive extrema is taken as the determination of the time scale for the intrinsic oscillation mode, since it not only provides a much higher resolution, but it can also be applied to the data with a non-zero mean (positive or negative values, without zero crossings). In our case, the axis of the channel numbers plays the role of the time axis.

The mode extraction method, called sifting, is described as follows [25]. The decomposition method uses envelopes built on local maxima and minima separately. For this purpose, the local extrema of the $f(t)$ signal are identified, and all maxima are interpolated by the cubic spline line as the upper envelope $U(t)$. In the same way, the lower spline envelope $L(t)$ is constructed on the minima. The mean between the upper and lower envelope $R(t)=(U(t)+L(t)) / 2$ receives the low-frequency residue status and is used for further transformations as $f(t)$, and the difference of the functions $f(t)$ and $R(t)$ receives the status of the first empirical mode $\varphi_{1}(t)$. 
The empirical mode is a function with the following properties: 1) the number of the function extrema on the interval considered differs by no more than one from the number of zero crossings of this function; 2) the half-sum of the upper and lower envelope of the function is close to zero.

As a result of the decomposition of the signal $f(t)$ into empirical modes, we get:

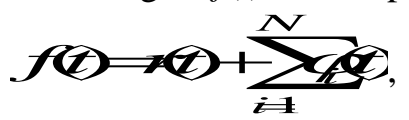

where $\varphi_{i}(t)$ are empirical modes, $r(t)$ is the trend residue. The first modes contain high-frequency components of the signal, and the last modes and the residue are low-frequency components.

\section{Fast decomposition algorithm}

We use the fast adaptive decomposition method proposed in [26]. Since we are considering a signature, the signal is one-dimensional: it depends only on the channel number. Accordingly, the algorithm is reduced to a one-dimensional version, and it is also simplified in comparison with [26] and [27] while preserving the idea of the original method [25]. Namely, the operations of constructing envelopes (upper and lower), and then, calculation of the points of the mean curve $R(i)$ between them, are replaced by smoothing (averaging) over a symmetric window of width $w$.

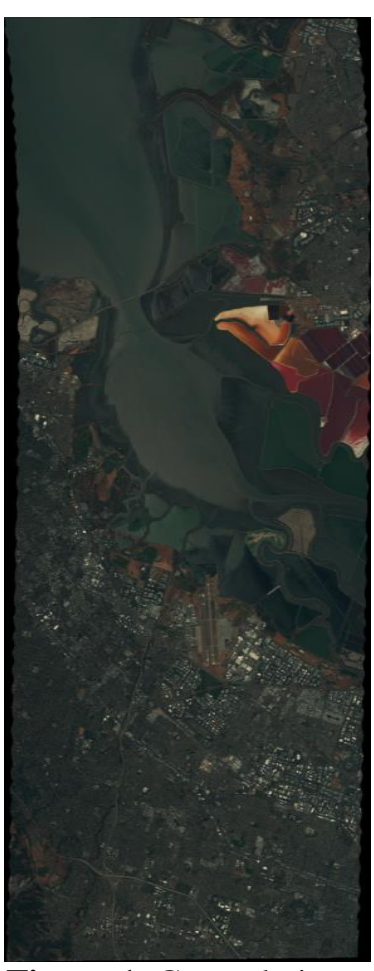

Figure 1. General view of the Moffett Field HIS.

\section{Algorithm steps:}

1. Assign the signature of the $k$-th pixel as the signal $f(i)$,

2. Initialize the size $w$ of the processing window with a value of 3 ( $w=$ $3)$, the number of the empirical mode is $q=0$.

3. Calculation of low-frequency residue

$$
R(i)=\frac{1}{w} \sum_{j=i-w / 2}^{i+w / 2} f(j),
$$

the values corresponding to the positions of the window that go beyond the limits of the signature are taken to be equal to the edge value of the signature.

4. Construct an empirical mode:

$$
q=q+1, \varphi_{q}(i)=f(i)-R(i)
$$

5. Find and enter into the arrays $p U$ and $p L$ all the points of local extrema (minima and maxima) of the current empirical mode $\varphi=\varphi_{q}$ within the window $W$ of width $w$ with the center in the current channel that satisfy the conditions:

$$
\begin{array}{ll}
\text { for the array } p U & \varphi(i)>\varphi(j), \forall j \in W_{w}(i), \\
\text { for the array } p L & \varphi(i)<\varphi(j), \forall j \in W_{w}(i),
\end{array}
$$

where $W_{w}(i)$ is a window of width $w$ with the center in the channel $i=$ $1, \ldots, n$.

6. If the number of maxima in $p U$ or the number of minima in $p L$ is less than 2, then $R$ cannot be further decomposed and the process terminates.

7. For each local maximum, find the distance $d_{\max }$ along the channel axis to another nearest maximum, for each local minimum, to another minimum $d_{\min }$ and then, take the smallest of them:

$$
d=\min \left(d_{\text {min }}, d_{\text {max }}\right) \text {, and update the window size } w=2\lceil d / 2\rceil+1
$$

8. Set $f(i)=R(i)$ and perform steps 3-7.

Figure 3 shows an example of signal decomposition into empirical modes.

\section{Experimental research}

The initial data are freely available hyperspectral images obtained with the use of the Airborne Visible/Infrared Imaging Spectrometer (AVIRIS). Figure 1 shows the general view of the Moffett 
Field HSI in three channels: 29th $-\mathrm{R}=638.2 \mathrm{~nm}$; 19 th $-\mathrm{G}=540.6 \mathrm{~nm}$; 11 th $-\mathrm{B}=462.8 \mathrm{~nm}$. This sample HSI shows various types of terrain, including water bodies, green vegetation and urban areas. As it was mentioned above, when constructing and analyzing compression algorithms for almost any data, the question arises about the presence of noisy data to which noise was added during the registration process or those data that were distorted as a result of transmission through the communication channels. The volume of such data can be significant and it can affect the resulting compression ratios. In some cases, information noise can be filtered in one way or another by data processing algorithms. Therefore, it is quite important to remove this noise before the compression of the original image. The chosen test sample is stored in the ENvironment for Visualizing Images format (ENVI) and has 2 bytes of information per pixel in each channel. Its width is 752 pixels, and its height is 1924 pixels. In total, the chosen HSI has 224 channels (about $10 \mathrm{~nm}$ wide each), which cover the wavelength range from $0.365 \mu \mathrm{m}$ to $2.497 \mu \mathrm{m}$. To investigate the correlation of images of adjacent HSI channels, a linear Pearson correlation coefficient was calculated between the current channel and the next one (223 pairs):

$$
r_{X Y}=\frac{\sum(X-\bar{X})(Y-\bar{Y})}{\sqrt{\sum(X-\bar{X})^{2} \sum(Y-\bar{Y})^{2}}} .
$$

The result of the calculation is shown in Table 1 .

Table 1. Distribution of the values of the Pearson correlation coefficient for adjacent HSI channels.

\begin{tabular}{c|cc}
\hline Range & Number of channels & In percentages, \% \\
\hline $0,9999-0,99999$ & 32 & 14.34 \\
$0,999-0,9999$ & 91 & 40.80 \\
$0,99-0,999$ & 48 & 21.52 \\
$0,9-0,99$ & 40 & 17.93 \\
$0,3-0,9$ & 12 & 5.83 \\
\hline
\end{tabular}

As can be seen from Table 1, over $94 \%$ of all channels have the correlation coefficient exceeding 0.9 , and over $76 \%$ of channels have the correlation coefficient exceeding 0.99 . This suggests that the neighboring channels are usually very similar to each other, despite their modulation by the influence of the atmosphere. Channels in which the correlation coefficient with the adjacent channel is lower than 0.99 , mainly fall within the wavelength ranges (near $1.4 \mu \mathrm{m}, 99-128$ channels of our HIS, and 1.9 $\mu \mathrm{m}, 153-166$ channels) of almost complete absorption of light by a mixture of water vapor and carbon dioxide. Approximately the same high absorption there is in the band above $2.5 \mu \mathrm{m}$ (channels 218 224). In this HSI it is about 50 channels (or $22 \%$ of this HSI), the contents of which are almost impossible to use because of the high noise level. If necessary, these channels can be deleted without loss to solve problems of classification of objects of the image.

\subsection{An example of a decomposition of a signature into empirical modes and low-frequency residuals.} Investigation of the possibilities

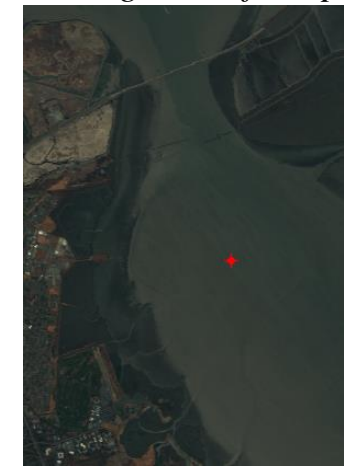

Figure 2. The pixel chosen for constructing empirical modes.
As standard for HSI storage is used 2 bytes per pixel per channel. The range of values of this type of data is significantly wider than that of universally used formats for storing visual information. One of an important reason for the introduction of the 2-byte accuracy of HSI is the high accuracy of modern spectrometers, which is achieved through special calibration methods [28]. At the same time, a fairly authoritative source [29] claims that already because of the influence of the atmosphere on the measurements of the spectrometer, it is difficult to talk about achieving accuracy higher than 2$5 \%$. This makes relevant the question of how many bits are needed to store one pixel in the HSI channel to exclude losses for future classification of HSI-objects, and to determine their state.

The application of the method of empirical modes will be considered using the water signature as an example. The pixel of the water sample is marked in red in Figure 2. Figure 4 shows an important for further analysis 
part of the result of the decomposition of the water signature into empirical modes and low-frequency residuals $(\mathrm{R})$. The complete decomposition of the signature contains 15 empirical modes and a trend.

Table 2 shows a number of dependencies on the number of the empirical mode (EM) for the first eight modes: 1) for the window size $w ; 2$ ) for the correlation coefficient of the original signature with its corresponding low-frequency residue; 3 ) for maximum deviation of the empirical mode from zero (Max) in absolute units of a 2-byte representation and in percentage to the maximum value of this signature and the maximum value throughout the HSI. Values in percent are given for comparison with an estimate of the achievable accuracy of HSI in 2-5\% given by D.Landgrebe (1999).

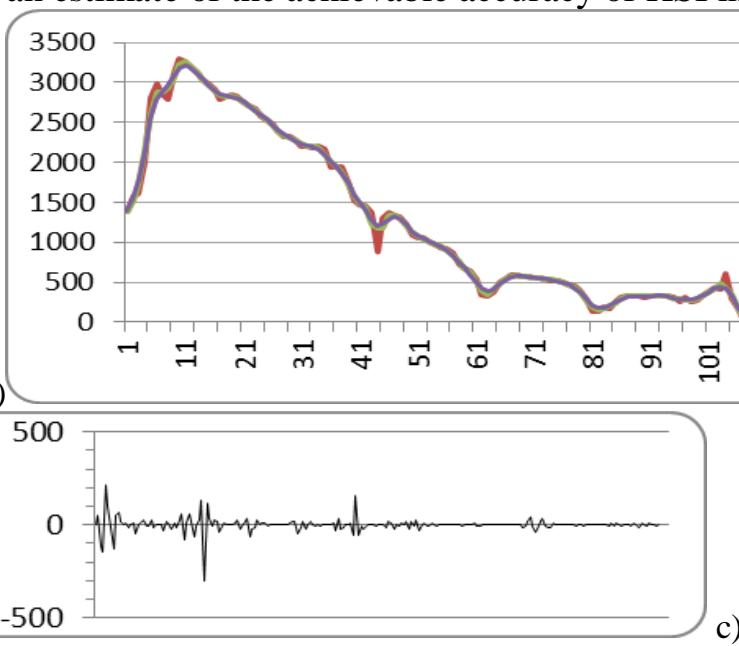

c) 1 .

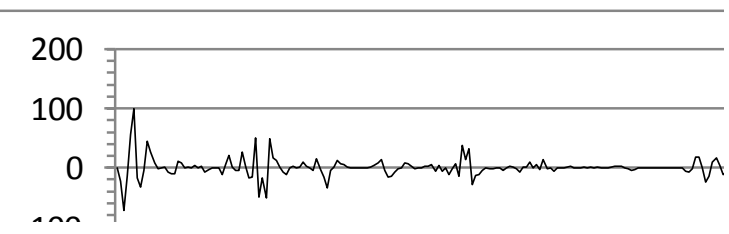

b)

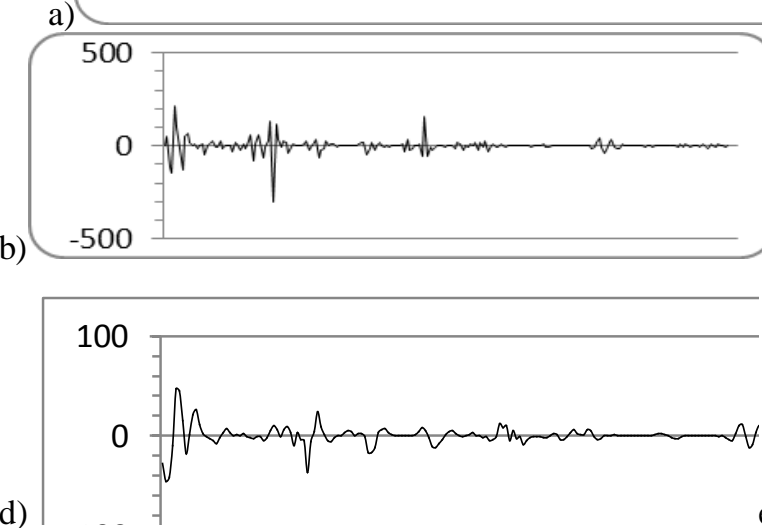

e)

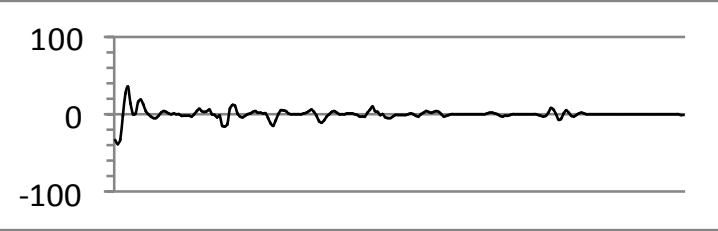

Figure 3. Decomposition of the signature into empirical modes and low-frequency residuals: a signature $(f)$, first (R1) and second (R2) low-frequency residuals; b-e - empirical modes 1-4.

The values of the Pearson correlation coefficient between signature and low-frequency residues are quite high: about from 0.999 to 0.99 (see table 2). Specifically, the significance of these values for the classification of HSI objects will be considered below, but on the whole this indicates that the signature in the correlation estimates can be replaced by at least its first low-frequency residues corresponding to the required accuracy.

Table 2. The change in the window size and in the maximum of the empirical mode (EM) with the its number up to 8 steps, the correlation coefficients of the signature $S$ with its low-frequency residuals R1-R8.

\begin{tabular}{|l|c|c|c|c|c|c|c|c|}
\hline Empirical Mode (EM) Number & 1 & 2 & 3 & 4 & 5 & 6 & 7 & 8 \\
\hline Window size $w$ & 3 & 3 & 3 & 3 & 3 & 3 & 7 & 10 \\
Correlation coefficient $r_{\mathrm{SR}}$ & 0,9991 & 0,9987 & 0,9982 & 0,9979 & 0,9976 & 0,9973 & 0,9950 & 0,9906 \\
Max of the EM & 306 & 99 & 47 & 36 & 26 & 21 & 267 & 215 \\
In [\%] to Max of the signature & 9.5 & 3.1 & 1.5 & 1.1 & 0.8 & 0.7 & 8.3 & 6.7 \\
In [\%] to Max of HSI & 4.1 & 1.3 & 0.6 & 0.5 & 0.3 & 0.3 & 3.6 & 2.9 \\
\hline
\end{tabular}

In the first 6 steps of the decomposition, the algorithm automatically selects a minimal blur window with a size of 3 channels. This may indicate the presence of noise, at least in part of the channels of our HSI. There are certain successes in correcting the influence of the atmosphere on the hyperspectral image [30], [31], but for channels where the signatures are close to zero, the correction is practically 
useless. From the point of view of the compression of the HSI, it is advantageous to detect noisy channels and remove them from consideration. Let's consider, whether assignment of the minimum size of the window can serve as the detector of noisiness of the channel.

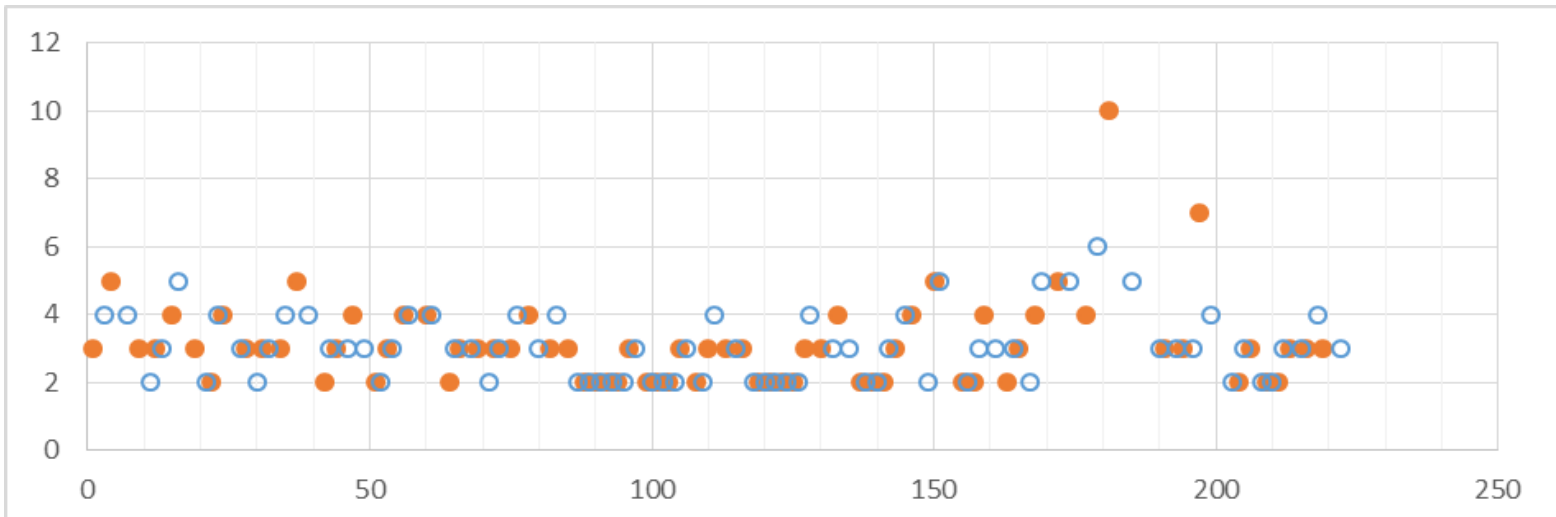

Figure 4. Distance (d) between adjacent extrema of the 1-st empirical mode: maxima (points with fill) and minima (point without fill), via the minimum of which the window size $w$ is determined. The horizontal axis is the channel number.

To do this, consider the distances between the current and next extremums (between two adjacent maxima, dmax, or minima, dmin), by which and according (6) the window size w is determined along

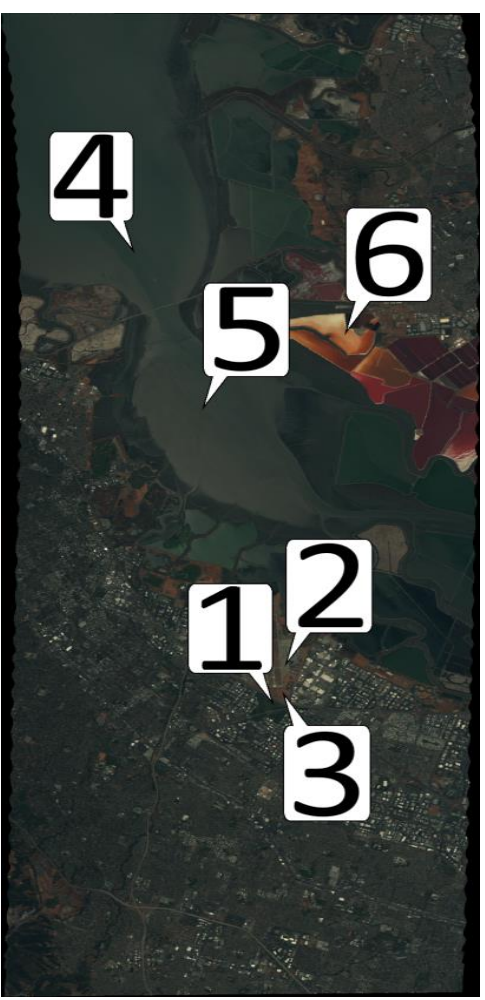

Figure 5. Samples of obiects for classifving. the axis of the channel numbers. The zone of noisy channels should give a smaller local window size than the local window size in the "clean" channels zone. The minimum possible value of $\mathrm{d}$, for which $\mathrm{w}=3$, is 2 . Figure 4 shows the distances between extrema for the first empirical mode.

As can be seen from the graph, the presence of the values $d=2$ is not limited to intervals 99-128, 153-166, although in these intervals they are the most. The appearance of $\mathrm{d}=2$ beyond the limits of these intervals correlates well with the windows of the influence of the atmosphere, but do not lead to a completely noisy channel. As a result, via the values of $\mathrm{d}=2$, we can only select the channels of suspects for noise and channels that are substantially modulated by the influence of the atmosphere.

3.2. Classification capabilities of signatures and their low-frequency residues on the basis of values of the correlation coefficient

As is known, each unique object of HSI has an individual signature. The difference between image objects is manifested in the difference in the behavior of signatures in some sequence of channels.

Let us consider the possibilities of classifying objects of the HSI via the signature of the object sample, using for this purpose different values of the Pearson correlation coefficient. Let us also consider how the transition to a 1-byte representation of the signature or the use of its low-frequency residuals instead of the signature will affect the classification capabilities. Let's select several pixels-samples of objects belonging to different objects (Figure 5). Sample 1 represents vegetation (tree crowns); sample 2 - concrete (from the airport runway); sample 3 - soil sample (Soil1) in the airport area; Samples 4 and 5 are water samples (Water1, Water2), but sample 4 looks like a darker one; sample 6 - soil sample (Soil2) from the field. Figure 7 shows the signatures that are constructed as sample averages from signatures that have a correlation coefficient with a sample signature of at least 0.999 . We calculate the correlation coefficient of the selected signature-samples with the signatures of all other pixels of the HSI. We shall establish the thresholds to be investigated for classifying an object based 
on the Pearson correlation coefficient with the sample signature: $\{0.9 ; 0.99 ; 0.999 ; 0.9999\}$. To the desired class we will include all the signatures that have a correlation coefficient with a sample greater than or equal to a given threshold. In addition to the binary classification (object / background) of the 2-byte representation of the HSI, a binary classification was performed on the first and second lowfrequency residue, as well as on the signature in a 1-byte representation. Then the difference in the classification results was calculated.

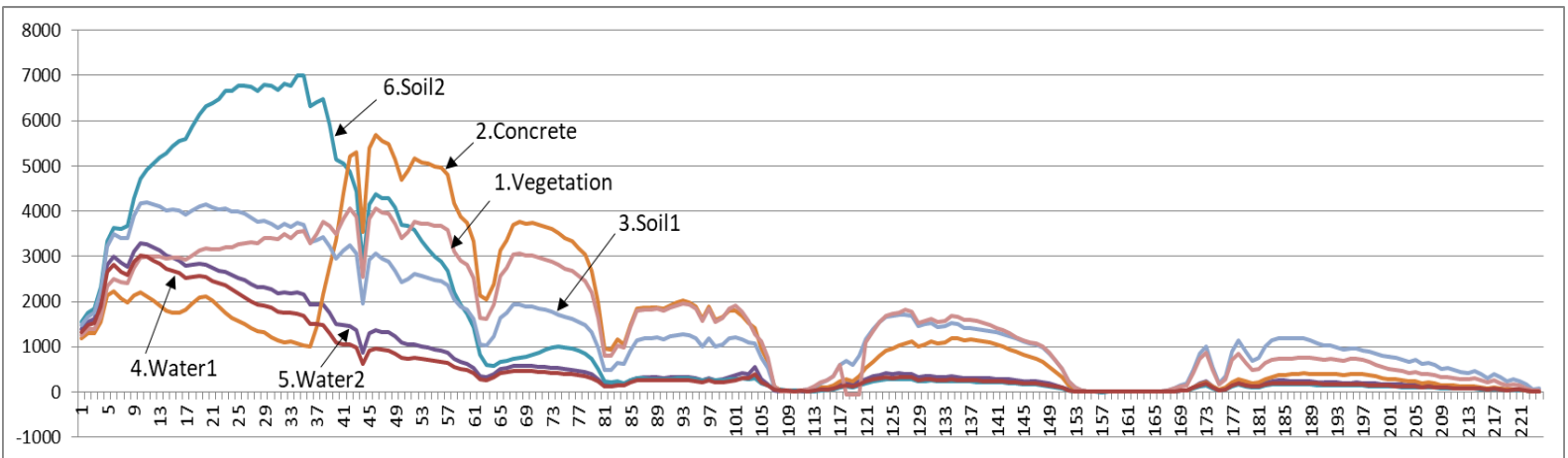

Figure 6. Signatures of samples of objects marked for classifying objects in Figure 5.

Examples of the classification of the initial HSI for different thresholds for the correlation coefficient are shown in Figure 7. The results of using in classifying the first or second low-frequency residue instead of the signature sample are shown in Table 3 (only for the 0.9 threshold). Comparative characteristics of the classification results for all signature samples in a 2-byte and 1-byte is given in Table 4 (only for the 0.999 threshold). These data show that the classification by the value of the correlation coefficient with signature sample can be used as a classification tool. In a number of cases, the threshold value of the correlation coefficient can play a generalizing role, as shown in Figure 7 (a and $\mathrm{c}$ ). The threshold $(\mathrm{T})$ of the correlation coefficient equal to 0.9 for the signature of sample 1 (Vegetation) made it possible to highlight almost the entire forest park and urban area, excluding building and road surface, and gave a classification mask that is practically complementary to the classification at $\mathrm{T}=0.9$ for sample 5 (Water2). The threshold $\mathrm{T}=0.9$ for sample 5 (Water2) captured besides water also the fields adjacent to the coastline, road surfaces, urban buildings. For the threshold $\mathrm{T}=0.99$ (Figure 7, b), we see in the Vegetation class only a very small number of objects. And at $\mathrm{T}=$ 0.999 - only 6 pixels corresponding to the sample. For sample 5 , Water 2 , the threshold $\mathrm{T}=0.99$ (Figure 7, d) gives already almost the entire surface of the water of the gulf and the flowing rivers, as well as the flood areas adjacent to the shoreline. The threshold $\mathrm{T}=0.999$ (Figure 7, e, f) already completely separates the objects specified by different samples of water 4 and 5 . The value of the threshold at 0.999 in all cases makes it possible to classify objects with signature shapes almost completely similar to the sample signature. The threshold value of 0.9999 guarantees the selection of objects completely identical to the sample or with the similar signature shapes, but allowing deviations in values not exceeding 2-5\%. Undoubtedly, the classification problem for the selected 6 samples can be solved on the basis of estimates [16]: $\Delta X_{i}, E\left(X_{i}\right), D\left(X_{i}\right)$, where $i$ is the channel number. Moreover, in the application of these estimations, which are intervalwise, one, two or three intervals will suffice, in which the signatures are clearly ranked by the value of the estimates.

Let us further consider the results of using, instead of the sample, the signature of its first or second low-frequency residue (see Table 3). Visually at $\mathrm{T}=0.9$ and $\mathrm{T}=0.99$, the difference in classification by signature and low-frequency residuals is observed as some smoothing and narrowing along the boundary of objects, increasing with increasing threshold. At $\mathrm{T}=0.99$, the detection density of the object area can also decrease (loss from 3\% density, to Water1, and up to 30\% - to Vegetation). At T = 0.999, significant losses occur in the object. 


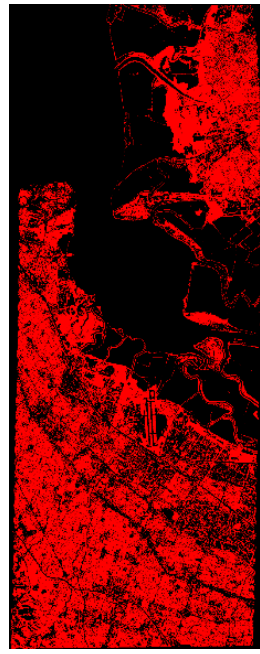

a

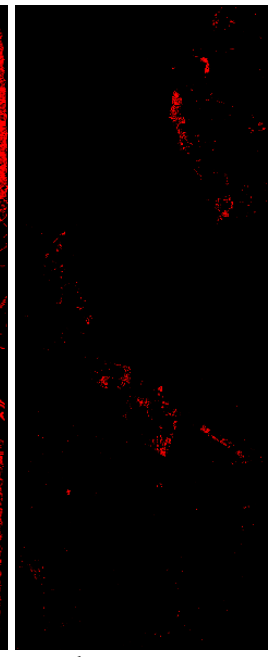

b

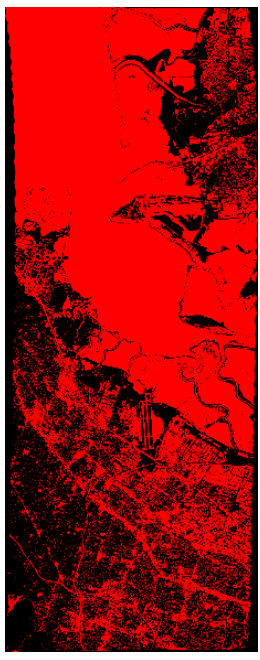

c

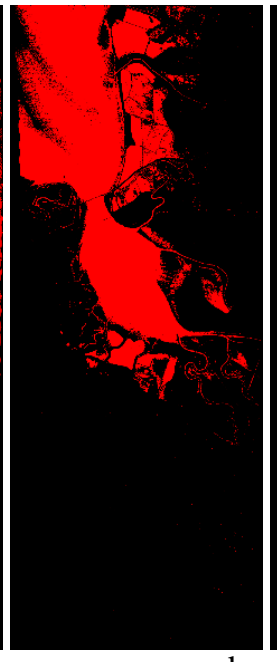

d

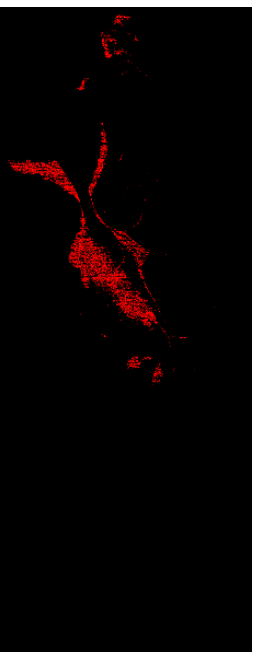

e

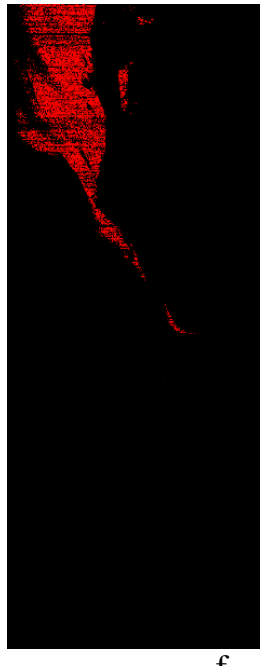

f

Figure 7. Examples of classification by object samples at different threshold values $(T)$ of the correlation coefficient: a) sample 1 - Vegetation, $\mathrm{T}=0.9$; b) sample 1 - Vegetation, $\mathrm{T}=0.99$; c) sample 5 - Water2, $\mathrm{T}=0.9$; d) sample 5 - Water2, $\mathrm{T}=0.99$; e) sample 5 - Water2, $\mathrm{T}=0.999$; f) sample 4 Water $1, \mathrm{~T}=0.999$.

Table 3. Classification of objects by the threshold of the correlation coefficient 0.9 for signatures of samples S in a 2-byte representation and their low-frequency residues R1 and R2.

\begin{tabular}{|c|c|c|c|c|c|c|c|c|c|}
\hline \multirow{3}{*}{$\mathrm{N}$} & \multirow{3}{*}{ Object } & \multirow{3}{*}{$\begin{array}{c}\text { Sample } \\
\text { coordinates } \\
(\mathrm{x} ; \mathrm{y})\end{array}$} & \multicolumn{3}{|c|}{ Pixels in Class } & \multicolumn{4}{|c|}{ Mismatched pixels } \\
\hline & & & \multirow{2}{*}{ via $S$} & \multirow{2}{*}{ via R1 } & \multirow{2}{*}{ via } & \multicolumn{2}{|c|}{ via R1 } & \multicolumn{2}{|c|}{ via $R 2$} \\
\hline & & & & & & pcs & $\%$ & pcs & $\%$ \\
\hline 1 & Vegetation & $442 ; 1332$ & 566744 & 558819 & 557542 & 8169 & 1.4 & 9846 & 1.7 \\
\hline 2 & Concrete & $447 ; 1275$ & 1018521 & 1014854 & 1015257 & 15303 & 1.5 & 21956 & 2.2 \\
\hline 3 & Soil1 & $426 ; 1351$ & 120603 & 116374 & 115699 & 4229 & 3.5 & 4904 & 4.1 \\
\hline 4 & Water1 & $209 ; 470$ & 767970 & 759931 & 756722 & 8061 & 1.0 & 11310 & 1.5 \\
\hline 5 & Water2 & $315 ; 775$ & 873712 & 864921 & 861536 & 8797 & 1.0 & 12206 & 1.4 \\
\hline 6 & Soil2 & $540 ; 630$ & 294865 & 283321 & 280976 & 11544 & 3.9 & 13889 & 4.7 \\
\hline
\end{tabular}

\subsection{Ability to switch from a 2-byte representation of signatures to a 1-byte}

The possibility of switching from a 2-byte representation of signatures to a 1-byte one was evaluated on the basis of a visual comparison of the results of a binary classification and a quantitative evaluation of the difference of the classification masks. A 1-byte representation is obtained from a 2byte division of double byte values by 32 with rounding. In all cases of visual comparison, the difference was not noticeable. The difference in masks in all cases remained within $7.5 \%$ (the worst case for the Water2 sample, see Table 4). The unmatched pixels in all cases were distributed over the area of the classified object in proportion to the density of the object.

Correlation coefficients between the signatures in the 2-byte and 1-byte representations for the samples: Vegetation, Concrete, Water1, Water2, Soil1, Soil2. Their values were 0.99997, 0.99998, $0.99994,0.99995,0.99998,0.99999$ respectively. The correlation coefficient decreases with increasing divisor. For example, for the Water2 sample, when the divisor is changed in the sequence $\{16,32$, $64\}$, the correlation coefficient with the 2-byte signature, while remaining high, changes as follows: 0.99998; 0.99995; 0.99980 .

This suggests that it is possible to switch to calculations with a 1-byte representation, if necessary, saving the divisor for backwards compatibility with a 2-byte representation. 
Table 4. Classification of objects at the correlation threshold of 0.999 for sample signatures in a 2byte and 1-byte representation.

\begin{tabular}{c|cccccc}
\hline \multirow{2}{*}{$\mathrm{N}$} & Object & $\begin{array}{c}\text { Sample } \\
\text { coordinates (x;y) }\end{array}$ & $\begin{array}{c}\text { Pixels in class } \\
\text { (2-byte) }\end{array}$ & $\begin{array}{c}\text { Pixels in class } \\
(1-\text { byte })\end{array}$ & \multicolumn{2}{c}{ Mismatched pixels } \\
\hline 1 & Vegetation & $442 ; 1332$ & 6 & 6 & pcs & $\%$ \\
2 & Concrete & $447 ; 1275$ & 430 & 429 & 7 & 0 \\
3 & Soil1 & $426 ; 1351$ & 920 & 904 & 20 & 1.6 \\
4 & Water1 & $209 ; 470$ & 60972 & 56418 & 4564 & 7.5 \\
5 & Water2 & $315 ; 775$ & 27176 & 26453 & 801 & 2.9 \\
6 & Soil2 & $540 ; 630$ & 831 & 826 & 5 & 0.6 \\
\hline
\end{tabular}

3.4. Improving the performance of calculations in the HSI classification and evaluating the possibility of the signature-based HSI compression

In order to improve the performance of the classification, an algorithm is implemented on the graphics processor. The working time of the classification algorithm based on the correlation coefficient with the sample signature is defined as the average (for 10 passes) total classification time for all 6 selected signatures. Instead of the sample signature, its low-frequency residue can be used. The measured classification time for the central (CPU) and graphics (GPU) processors is shown in Table 5.

Table 5. Performance achieved on CPU and GPU for HSI classification via value of correlation coefficient.

\begin{tabular}{l|ccc}
\hline Hardware & Number of Core & Clock frequency, MHz & Time (sec) \\
\hline CPU: AMD Phenom II X4 955 & 4 & 2500 & 257.07 \\
GPU: NVIDIA GeForce GTX 970 & 1664 (CUDA) & 1152 & 0.31 \\
\hline
\end{tabular}

From Table 5, it can be seen that the use of a graphics processor makes it possible to carry out the classification procedure 836 times ( 3 orders of magnitude) faster than on a central processor. In this case, we can talk about the operation of the algorithm in real time, which makes its use in desktop data processing applications quite realistic. The performance for the same CPU of the empirical mode decomposition was 0.91 seconds per thousand signatures.

In the interest of evaluating the capabilities of signature-based compression, an attempt is made to increase the number of zero or close to zero values in a compressible array by applying wavelet decomposition. Based on the length of the signature array, 7 steps of the Haar transformation are used for the signature wavelet decomposition of the HSI in the 1-byte representations. After that, the source 2-byte HSI and the result of the Haar transformation were archived by ZIP (without losses) with the maximum compression ratio (Table 6). The compression ratio for 1-byte signatures was 6.65. Since we replaced the double-byte representation with a 1-byte representation, we will treat this compression as nearlossless.

Table 6. Compression of the entire HSI data array.

\begin{tabular}{ccc}
\hline File size, MB & Without wavelet decomposition & 1-byte signature with wavelet decomposition \\
\hline Source file & 648,18 & 324,09 \\
Archived file & 421,12 & 97,48 \\
\hline
\end{tabular}

\section{Conclusion}

The signature of hyperspectral image (HSI) pixels and their decomposition into empirical modes (EM) and low-frequency residuals are investigated. On the basis of estimates related to the EMdecomposition method, the possibility of switching from a 2-byte representation of the values of the HIS-signature to a 1-byte one is examined using the example of the Moffett Field from the AVIRIS spectrometer.

It is revealed that: 1) the localization of the minimum window sizes for the first EM is correlated with the localization of the significant influence of the atmosphere; 2) the first low-frequency residues have a fairly high correlation coefficient with the signature; 3) the greatest interest for the 
decomposition of the signature is represented by one or two first EM and the corresponding lowfrequency residue; 4) the 1 st and 2 nd modes on the significant part of the channel axis are close to zero and can be reduced to a 1-byte representation practically without loss of accuracy; 5) 50 of the 224 HIS-channels are noisy and can be excluded from consideration.

The management of the classification capabilities of signatures by changing the threshold value of the correlation coefficient with the sample, as well as the application of the 1st and 2nd low-frequency residues in place of the signature, was studied. Classification capabilities of signatures in a 1-byte representation are almost equivalent to a 2-byte one, which makes it possible to put a signature with 1byte representation (as eight senior digits) as the object of compression.

The classification procedure is implemented on the GPU, which accelerated its execution more than 800 times, to fractions of a second.

Classification according to the samples using [16] on the basis of estimates $\Delta X_{i}, E\left(X_{i}\right), D\left(X_{i}\right)$, where $i$ is the channel number, can additionally reduce the number of channels necessary for classification. If the estimates are applied in a series of channel intervals, one or three intervals may be sufficient, in which the signatures are clearly ranked by the values of the estimates.

For the wavelet decomposition of the HSI data array, in combination with a 1-byte representation, a nearlossless compression ratio of 6.65 is obtained.

For the future works it is interesting to investigate also the approaches based on the machine learning methods like publications [19],[33],[34],[35].

\section{References}

[1] Wu X and Memon N 1997 Context-based, adaptive, lossless image coding IEEE Trans. Commun. Apr. 45 437-444

[2] Weinberger M J, Seroussi G and Sapiro G 2000 The LOCO-I lossless image compression algorithm: Principles and standardization into JPEG-LS IEEE Trans. Image Processing 9 13091324

[3] Wu X and Memon N 2000 Context-based lossless interband compression - Extending CALIC IEEE Trans. Image Processing 9 994-1001

[4] Magli E, Olmo G and Quacchio E 2004 Optimized onboard lossless and near-lossless compression of hyperspectral data using CALIC IEEE Geoscience and Remote Sensing Letters 1(1) $21-25$

[5] Wu X, Memon N and Sayood K 1995 A Context-Based, Adaptive, Lossless/Nearly-Lossless Coding Scheme for Continuous-Tone Images ISO/IEC JTC 1/SC 29/WC 1202

[6] Kozoderov V V, Kondranin T V, Kazancev O, Bobylev V I, Scherbakov M V, Borzyak V V, Dmitriev E V, Egorov V D, Kamencev V P, Belyakov A Yu and Loginov S B 2009 Processing and interpretation of hyperspectral aerospace measurement data for remote diagnostics of natural and technogenic objects Earth Research from Space 2 36-54

[7] Chaban L N, Vecheruk G V and T S 2009 Gavrilova. Investigation of the possibilities of land cover classification using hyperspectral imagery in thematic processing packages of remote sensing data Proceedings of MIPT 1(3) 171-180

[8] Chaban L N, Vecheruk G V, Kondranin T V, Kudryavtsev S V and Nikolenko A A 2012 Modeling and thematic processing of images identical to video data of ERS hyperspectral equipment under development Current Problems of Earth Remote Sensing from Space 9(2) 111121

[9] Popov M A and Stankevich S A 2006 Methods for optimizing the number of spectral channels in problems of ERS data processing and analysis Contemporary Problems of Earth Remote Sensing from Space 1 106-112

[10] Jiménez L and Landgrebe D A 1999 Supervised Classification in High Dimensional Space: Geometrical, Statistical, and Asymptotical Properties of Multivariate Data IEEE Transactions on Systems, Man, And Cybernetics-Part C: Applications and Reviews 28(1) 39-53 
[11] Arzuaga-Cruz E, Jimenez-Rodriguez L O and Velez-Reyes M 2003 Unsupervised Feature Extraction and Band Subset Selection Techniques Based on Relative Entropy Criteria for Hyperspectral Data Analysis Proc. SPIE 5093 462-473

[12] Lee C and Langrebe D A 1993 Feature Extraction Based on Decision Boundaries IEEE Trans. on Pattern Analysis and Machine Intelligence 4(15) 388-400

[13] Myasnikov E V 2017 Hyperspectral image segmentation using dimensionality reduction and classical segmentation approaches Computer Optics 41(4) 564-572 DOI: 10.18287/2412-61792017-41-4-564-572

[14] Nakariyakul S and Casasent D 2004 Hyperspectral feature selection and fusion for detection of chicken skin tumors Proc. SPIE 5271 128-139 DOI: 10.1117/12.517443

[15] Zamyatin A V and Sarinova A Zh 2013 An algorithm for compressing hyperspectral aerospace images with the account of inter-band correlation Applied Informatics 5(47) 35-42

[16] Gashnikov M V and Glumov N I 2014 Hierarchical compression for hyperspectral image storage Computer Optics 38(3) 482-488

[17] Gashnikov M V and Glumov N I 2016 Onboard processing of hyperspectral data in the remote sensing systems based on hierarchical compression Computer Optics 40(4) 543-551 DOI: 10.18287/2412-6179-2016-40-4-543-551

[18] Gashnikov M V, Glumov N I, Kuznetsov A V, Mitekin V A, Myasnikov V V and Sergeev V V 2016 Hyperspectral remote sensing data compression and protection Computer Optics 40(5) 689-712 DOI: 10.18287/2412-6179-2016-40-5-689-712

[19] Vorobiova N S, Sergeyev V V and Chernov AV 2016 Information technology of early crop identification by using satellite images Computer Optics 40(6) 929-938 DOI: 10.18287/24126179-2016-40-6-929-938

[20] Auge E, S'anchez J E, Kiely A, Blanes I and Serra-Sagrista J 2013 Performance impact of parameter tuning on the CCSDS-123 lossless multi- and hyperspectral image compression standard Journal of Applied Remote Sensing 7(1) 16

[21] Blanes I, Magli E and Serra-Sagrisat J 2014 A tutorial on Image Compression on Optical Space Imaging Systems IEEE Geoscience and Remote Sensing Magazine 2(3) 8-26

[22] Abramov S K and Lukin V V 2015 Problems of automating the processing of hyperspectral remote sensing images Aerospace Engineering and Technology 6(123) 101-110

[23] Abramova V V, Abramov S K and Lukin V V 2015 Multistage Iterative Method for Blind Evaluation of Mixed Noise Characteristics on Images ITS 6(1) 8-14

[24] Lukin V, Abramov S, Ponomarenko N, Uss M, Zriakhov M, Vozel B, Chehdi K and Astola J 2011 Methods and automatic procedures for processing images based on blind evaluation of noise type and characteristics SPIE Journal of Applied Remote Sensing 5(1) 27 DOI: $10.1117 / 1.3539768$

[25] Huang N E, Shen Z, Long S, Wu M C, Shih H H, Zheng Q, Yen N-C, Tung C C and Liu H H 1998 The Empirical Mode Decomposition and Hilbert Spectrum for nonlinear and nonstationary time series analysis Proc. R. Soc. London A $454903-995$

[26] Bhuiyan S M A, Adhami R R and Khan J F 2008 A novel approach of fast and adaptive bidimensional empirical mode decomposition IEEE International Conference on Acoustics, Speech and Signal Processing 1313-1316

[27] Guryanov F and Krylov A 2017 Fast medical image registration using bidirectional empirical mode decomposition Signal Processing: Image Communication 1-6

[28] Introduction to Hyperspectral Imaging: Tutorial MicroImages Inc. (Access mode: http://www.microimages.com/documentation/Tutorials/hyprspec.pdf)

[29] Podlipnov V V and Skidanov R V 2017 Calibration of an imaging hyperspectrometer Computer Optics 41(6) 869-874

[30] Landgrebe D 1999 Information Extraction Principles and Methods for Multispectral and Hyperspectral Image Data Information Processing for Remote Sensing 3-38

[31] Denisova A Y and Myasnikov V V 2016 Atmospheric correction of hyperspectral images using small volume of the verified data Computer Optics 40(4) 526-534 DOI: 10.18287/2412-61792016-40-1-526-534 
[32] Denisova A Y, Juravel Y N and Myasnikov V V 2016 Estimation of parameters of a linear spectral mixture for hyperspectral images with atmospheric distortions Computer Optics 40(3) 380-387 DOI: 10.18287/2412-6179-2016-40-3-380-387

[33] Sirota A A and Dryuchenko M A 2015 Generalized image compression algorithms for arbitrarily-shaped fragments and their implementation using artificial neural networks Computer Optics 39(5) 751-761 DOI: 10.18287/0134-2452-2015-39-5-751-761

[34] Sergeev V V and Yuzkiv R R 2016 A parametric model for the autocorrelation function of space hyperspectral data Computer Optics 40(3) 416-421 DOI: 10.18287/0134-2452-2016-40-3-416421

[35] Kopenkov V N and Myasnikov V V 2016 Development of an algorithm for automatic construction of a computational procedure of local image processing, based on the hierarchical regression Computer Optics 40(5) 713-720 DOI: 10.18287/2412-6179-2016-40-5-713-720

\section{Acknowledgements}

This work was supported by a grant from the Russian Science Foundation (project No. 16-11-00068). 